\title{
Torsional Rigidity of Minimal Submanifolds
}

\author{
Markvorsen, Steen; Palmer, Vicente
}

Published in:

Proceedings of the London Mathematical Society

Link to article, DOI:

10.1112/S0024611505015716

Publication date:

2006

Document Version

Publisher's PDF, also known as Version of record

Link back to DTU Orbit

Citation (APA):

Markvorsen, S., \& Palmer, V. (2006). Torsional Rigidity of Minimal Submanifolds. Proceedings of the London Mathematical Society, 93(3), 253-272. https://doi.org/10.1112/S0024611505015716

\section{General rights}

Copyright and moral rights for the publications made accessible in the public portal are retained by the authors and/or other copyright owners and it is a condition of accessing publications that users recognise and abide by the legal requirements associated with these rights.

- Users may download and print one copy of any publication from the public portal for the purpose of private study or research.

- You may not further distribute the material or use it for any profit-making activity or commercial gain

- You may freely distribute the URL identifying the publication in the public portal

If you believe that this document breaches copyright please contact us providing details, and we will remove access to the work immediately and investigate your claim. 


\title{
TORSIONAL RIGIDITY OF MINIMAL SUBMANIFOLDS
}

\author{
STEEN MARKVORSEN\# AND VICENTE PALMER*
}

\begin{abstract}
We prove explicit upper bounds for the torsional rigidity of extrinsic domains of minimal submanifolds $P^{m}$ in ambient Riemannian manifolds $N^{n}$ with a pole $p$. The upper bounds are given in terms of the torsional rigidities of corresponding Schwarz symmetrizations of the domains in warped product model spaces. Our main results are obtained via previously established isoperimetric inequalities, which are here extended to hold for this more general setting based on warped product comparison spaces. We also characterize the geometry of those situations in which the upper bounds for the torsional rigidity are actually attained and give conditions under which the geometric average of the stochastic mean exit time for Brownian motion at infinity is finite.
\end{abstract}

\section{Introduction}

We consider a complete Riemannian manifold $\left(M^{n}, g\right)$ and the induced Brownian motion $X_{t}$ defined on $M$. The analytic moments of exit time of $X_{t}$ from precompact domains $D$ in the manifold are given

2000 Mathematics Subject Classification. Primary 53C, 35K, 60J.

Key words and phrases. Minimal submanifolds, extrinsic balls, torsional rigidity, mean exit time, isoperimetric inequalities, Faber-Krahn inequalities, Schwarz symmetrization.

\#Work partially supported by the Danish Natural Science Research Council and DGI grant MTM2004-06015-C02-02.

* Work partially supported by the Caixa Castelló Foundation, DGI grant MTM2004-06015-C02-02, and by the Danish Natural Science Research Council. 
by the integrals

$$
\mathcal{A}_{p, k}(D)=\int_{D} u_{k}^{p}(x) d \mu
$$

The functions $u_{k}$ are defined inductively as the sequence of solutions to the following hierarchy of boundary value problems (see [Mc], and $[\mathrm{H}])$ :

$$
\begin{gathered}
\Delta^{M} u_{1}+1=0 \text { on } D \\
\left.u_{1}\right|_{\partial D}=0,
\end{gathered}
$$

and, for $k \geq 2$,

$$
\Delta^{M} u_{k}+k u_{k-1}=0 \text { on } D
$$

$$
\left.u_{k}\right|_{\partial D}=0
$$

Here $\Delta^{M}$ denotes the Laplace-Beltrami operator on $\left(M^{n}, g\right)$. The first solution $u_{1}(x)$ in this hierarchy is the mean exit time of first exit from $D$ for a Brownian particle starting at the point $x$ in $D$, (see [Dy]). Since this function will be given special attention in this paper, we shall denote it $u_{1}=E$. The sequence (1.1) is the so-called $L^{p}$-moment spectrum of the domain $D$ (see $[\mathrm{Mc}])$. In close analogy with the Dirichlet spectrum, the cases $p=1$ and $p=\infty$ are of special interest. Following [Mc] we write

$$
\mathcal{A}_{k}(D)=\mathcal{A}_{1, k}(D)
$$

The quantity $\mathcal{A}_{1}(D)$ is known as the torsional rigidity of $D$, because when $D \subseteq \mathbb{R}^{2}, \mathcal{A}_{1}(D)$ represents the torque required for a unit angle of twist per unit length when twisting an elastic beam of uniform cross section $D$, (see $[\mathrm{Ba}]$ and $[\mathrm{PS}])$. The torsional rigidity $\mathcal{A}_{1}(D)$ plays a role 
in the spectrum (1.4) which is similar to the role of the first positive Dirichlet eigenvalue in the Dirichlet spectrum. There are a number of works which deal with bounds for the first Dirichlet eigenvalue and for the torsional rigidity in terms of geometric invariants associated with the underlying domain. See e.g. [Ber], [Ch1], [Ch2] concerning the first Dirichlet eigenvalue, and $[\mathrm{Ba}],[\mathrm{PS}],[\mathrm{BBC}]$, and $[\mathrm{BG}]$ concerning the torsional rigidity.

Perhaps the most relevant example and token of interest in these problems is given by the St. Venant torsion problem. It is a precise analog of the Rayleigh conjecture about the fundamental tone of a membrane. In 1856, Saint-Venant conjectured that of all cross sections with a given area, the circle has maximum torsional rigidity. The first proof of this conjecture was given by G. Pólya in 1948, (see [Po] and also $[\mathrm{PS}]$ ), using the method of symmetrizations due to J. Steiner.

The proof of the Rayleigh conjecture in the context of Riemannian geometry is based on the Faber-Krahn inequality which in turn is based on the standard isoperimetric inequality for domains. This inequality thereby provides the connection between the bounds for the first Dirichlet eigenvalue and the geometry of the underlying Riemannian manifold in which the actual domain is residing.

In the paper [Mc], P. McDonald combines both techniques, using now Schwarz symmetrization and Faber-Krahn's inequality, in order to establish relations between the $L^{1}$-moment spectrum of a domain 
and the geometry of the underlying manifold. He thereby obtains upper bounds for the $L^{1}$-moment spectrum of smoothly bounded domains with compact closure in a Riemannian manifold provided that all the domains in the manifold satisfies a certain type of isoperimetric conditions. The isoperimetric conditions are formulated in comparison with the real space forms of constant curvature $\mathbb{K}^{n}(b), b \in \mathbb{R}$ (see Theorem 1.2 in $[\mathrm{Mc}])$.

The purpose of the present work is to exploit - in this vein - the torsional rigidity of some specific domains of minimal submanifolds in ambient spaces with suitably bounded curvature. We do not need explicit isoperimetric conditions on the domains as in [Mc], but construct more flexible comparison spaces, namely warped products which are specially prepared to estimate the torsional rigidity of the domains in question. One particular property needed to be satisfied in these comparison spaces is a two-sided isoperimetric inequality type condition which we call a balance condition.

The geometrical analysis of the extrinsic distance function has been used in several works (see [CLY], [Ma1], [Ma2], [MP1], [MP2], [MP3]) to study the influence of the extrinsic curvatures of a submanifold and the intrinsic curvatures of the ambient manifold on the behavior of the solutions of certain classical PDEs. The extrinsic $R$-balls $D_{R}$ (which, roughly speaking, are defined as the intersections of the geodesic balls $B_{R}^{n}$ in the ambient space, with the submanifold in question), has in this setting turned out to be a fundamental tool for the description of the relationship between the inner and the outer geometry of immersed 
submanifolds in ambient Riemannian manifolds.

In the present paper, Theorem 2.1 gives an upper bound for the torsional rigidity of extrinsic $p$-centered $R$-balls of a minimal submanifold $P^{m}$ in an ambient complete non-compact Riemannian manifold $N^{n}$, which admits $p$ as a pole. We assume throughout that $p \in P$, but recall also that, considered as a pole in the ambient space $N$, the exponential map from there is a diffeomorphism: $\exp _{p}: T_{p} N^{n} \rightarrow N^{n}$. For comparison, an Hadamard-Cartan manifold has everywhere nonpositive sectional curvatures and since it is also by definition simply connected, every point is a pole. The rôle of the pole $p$ is precisely to serve as the origin of a smooth distance function $\operatorname{dist}_{N}(p, x)$ from $p$ : For every $x \in N^{n}-\{p\}$ we define $r(x)=\operatorname{dist}_{N}(p, x)$, and this distance is realized by a unique geodesic from $p$ to $x$. The sectional curvatures of $N$ along these geodesics are called the $p$-radial sectional curvatures of $N$ and are denoted by $K_{p, N}(x)$.

Throughout this paper we shall assume that the ambient manifold $N^{n}$ has its $p$-radial sectional curvatures $K_{p, N}(x)$ bounded from above by the expression $-w^{\prime \prime}(r(x)) / w(r(x))$, which are precisely the radial sectional curvatures of a so-called $w$-model space $M_{w}^{m}$. Such a model space is defined as the warped product of a real interval with the standard $(m-1)$-dimensional unit Euclidean sphere $S_{1}^{m-1}$ and warping function $w(r)$ which satisfies the initial conditions $w(0)=0, w^{\prime}(0)=1$. A precise definition and further properties of model spaces will be given in section 3 below. 
For now we only need to observe, that the following conditions and concepts will be instrumental for our results and for their initial formulation.

1.1. Comparison constellations. The setting of our comparison strategy is contained in the following definition:

Definition 1.1. The triple $\left\{N^{n}, P^{m}, M_{w}^{m}\right\}$, consisting of an ambient space $N^{n}$, a minimal submanifold $P^{m} \subset N^{n}$ and a comparison $w$-model space $M_{w}^{m}$ which satisfy the assumptions indicated above, will be called a comparison constellation.

Specifically, in a comparison constellation we thus assume, that there is a point $p$ in $P$ which is also a pole of the ambient space $N$ and that the radial sectional curvatures as seen from $p$ satisfy $K_{p, N}(x) \leq$ $K_{w}(r(x))=-w^{\prime \prime}(r) / w(r)$ for all $r \leq R$, where $R$ is the radius of any extrinsic $p$-centered $R$-ball $D_{R}$ under consideration in $P$. We will pay special attention to the intrinsic geometry of the $w$-model spaces. It is important to note, that the warping function $w(r)$ for the comparison space $M_{w}^{m}$ must satisfy the 'smooth pole' conditions $w(0)=0$ and $w^{\prime}(0)=1$ and that for symmetrization purposes we shall be using the model spaces beyond the radius $R$. In particular we shall assume that the so-called balancing conditions are satisfied for all $r$.

1.2. Balanced model spaces. At the outset the $w$-model spaces are completely determined via $w$ by the mean curvatures of the spherical fibers $S_{r}^{w}$ - at distance $r$ from the center point of the model space the fiber mean curvature is

$$
\eta_{w}(r)=w^{\prime}(r) / w(r)
$$


by the volume of the fiber

$$
\operatorname{Vol}\left(S_{r}^{w}\right)=V_{0} w^{m-1}(r)
$$

and by the volume of the corresponding ball, for which the fiber is the boundary

$$
\operatorname{Vol}\left(B_{r}^{w}\right)=V_{0} \int_{0}^{r} w^{m-1}(t) d t
$$

Here $V_{0}$ denotes the volume of the unit sphere $S_{1}^{m-1}$. The latter two functions define the isoperimetric quotient function as follows

$$
q_{w}(r)=\operatorname{Vol}\left(B_{r}^{w}\right) / \operatorname{Vol}\left(S_{r}^{w}\right)
$$

As mentioned previously, the model spaces serve foremost as comparison controllers for the radial sectional curvatures of $N^{n}$, but we shall need two further purely intrinsic conditions on the model spaces: A given $w$-model space $M_{w}^{m}$ is called balanced from below and balanced from above, respectively, if the following weighted isoperimetric conditions are satisfied:

Balance from below: $q_{w}(r) \eta_{w}(r) \geq 1 / m$ for all $r \geq 0$;

Balance from above: $q_{w}(r) \eta_{w}(r) \leq 1 /(m-1)$ for all $r \geq 0$. A model space is called totally balanced if it is balanced both from below and from above. See examples 3.13.

1.3. The key Jacobi field technique. The curvatures of the ambient space $N$ control the second order behavior of $r(x)$ via the classical Jacobi field index theory, see e.g. [S]. A bound on the $p$-radial sectional curvatures thus gives a bound on the Hessian of radial functions, 
$\operatorname{Hess}^{N}(f(r))$, as proved by Greene and Wu, see [GreW] and applied by Jorge and Koutroufiotis, [JK]. These techniques may be considered as generalizations (to submanifolds) of purely intrinsic comparison methods - an observation which motivates our corollary 2.4, where we give an upper bound for the torsional rigidity of geodesic balls in a manifold $N$ with a pole $p$, and $p$-radial sectional curvatures bounded from above, by specializing the proof of Theorem 2.1 to this purely intrinsic setting, where $P=N$. In this case, the extrinsic geometry disappears from the analysis and only the intrinsic geometry of $N$ is active.

\section{Main Results}

Although the remarks and tools stated above will be substantially refined in the following sections, so far they do permit the statement of our main theorem:

Theorem 2.1 (Main result). Let $\left\{N^{n}, P^{m}, M_{w}^{m}\right\}$ denote a comparison constellation, and assume that $M_{w}^{m}$ is totally balanced. Let $D_{R}$ be a precompact extrinsic $R$-ball in $P^{m}$, with center at a point $p \in P$ which also serves as a pole in $N$. Then

$$
\mathcal{A}_{1}\left(D_{R}\right) \leq \mathcal{A}_{1}\left(B_{S(R)}^{w}\right)
$$

where $B_{S(R)}^{w}$ is the Schwarz symmetrization of $D_{R}$ in the $w$-model space $M_{w}^{m}$, i.e., it is the geodesic ball in $M_{w}^{m}$ such that $\operatorname{Vol}\left(D_{R}\right)=\operatorname{Vol}\left(B_{S(R)}^{w}\right)$. Equality in (2.1) for some fixed radius $R$ implies that $D_{R}$ is a minimal cone in $P^{m}$, and if the ambient manifold is the real space form $\mathbb{K}^{n}(b)$ with constant sectional curvature $b<0$, this equality implies that $P^{m}$ is totally geodesic in $\mathbb{K}^{n}(b)$. 
Remark 2.2. By definition, the torsional rigidity $\mathcal{A}_{1}\left(D_{R}\right)$ is the $D(R)$ integral of the mean exit time function $E_{R}(x)$ from $x$ in $D_{R}$. We note that for most minimally immersed submanifolds $P^{m}$ in the flat Euclidean spaces $\mathbb{R}^{n}$ with the obvious choice of comparison model space, $M_{w}^{m}=\mathbb{R}^{m}, w(r)=r$, we have (see [Ma1], [Pa]):

$$
\begin{aligned}
& E_{R}(x)=E_{R}^{w}(r(x)) \text { for all } x \in D_{R}, \\
& \text { but also } \operatorname{Vol}\left(D_{R}\right)>\operatorname{Vol}\left(B_{R}^{w}\right) \\
& \quad \text { so that } \mathcal{A}_{1}\left(D_{R}\right)>\mathcal{A}_{1}\left(B_{R}^{w}\right)
\end{aligned}
$$

This shows in particular the powers of our symmetrization techniques for obtaining general upper bounds for the torsional rigidity as in Theorem 2.1, equation (2.1).

The geometric average mean exit time from $D(R)$ is given by the quotient $\mathcal{A}_{1}\left(D_{R}\right) / \operatorname{Vol}\left(D_{R}\right)$. As will be observed below in corollary 5.2 this quotient is unbounded for geodesic balls in all Euclidean spaces as $R \rightarrow \infty$. The existence of regions in $\mathbb{R}^{m}$ with finite torsional rigidity and yet infinite volume follows from the work $[\mathrm{BBC}]$. In $[\mathrm{BG}]$ the authors find further upper bounds for the torsional rigidity of domains in complete non-compact manifolds. In both cases the bounds are obtained from Hardy inequalities, which, geometrically speaking, guarantees that the boundaries of the domains under consideration are not too thin - so that the diffusion is thus guaranteed sufficient room for escape. In our present setting this property is obtained partly from the constructive simplicity of the submanifold domains, that we consider, and partly from the isoperimetric type balancing conditions of 
the comparison spaces, that we use.

Although Brownian diffusion is known to be transient in Euclidean spaces of dimensions larger than 2, it is not sufficiently swift, however, to give even a finite average of the mean exit time at infinity for geodesic balls. However, the situation is different in spaces with sufficiently controlled curvatures as we demonstrate in the following corollary - to be proved in section 5 .

Corollary 2.3. Let $\left\{N^{n}, P^{m}, M_{w}^{m}\right\}$ denote a comparison constellation. Assume that the extrinsic balls $D_{R}$ in $P^{m}$ are well-defined and precompact for all $R$. Suppose that the model space $M_{w}^{m}$ in the constellation is totally balanced and suppose further that the model space geodesic spheres do not have 0 as a limit for their mean curvatures $\eta_{w}(r)$ as $r \rightarrow \infty$. Then the limit $q_{w}(\infty)$ of the isoperimetric quotient function is finite and it bounds the average mean exit time at infinity in $P^{m}$ in the following sense:

$$
\lim _{R \rightarrow \infty}\left(\frac{\mathcal{A}_{1}\left(D_{R}\right)}{\operatorname{Vol}\left(D_{R}\right)}\right) \leq q_{w}^{2}(\infty)
$$

By specializing the proof of Theorem 2.1 to the purely intrinsic geometric setting (so that the submanifold is the ambient manifold itself, $P=N$ ), we obtain the following

Corollary 2.4. Let $B_{R}^{n}=D_{R}$ be a geodesic ball of a complete manifold $N^{n}$ with a pole $p$, and let $\left\{N^{n}, N^{n}, M_{w}^{n}\right\}$ denote a corresponding comparison constellation with an $n$-dimensional $w$-model space $M_{w}^{n}$ which is totally balanced. Then

$$
\mathcal{A}_{1}\left(B_{R}^{n}\right) \leq \mathcal{A}_{1}\left(B_{S(R)}^{w}\right)
$$


where $B_{S(R)}^{w}$ is the Schwarz Symmetrization of $B_{R}^{n}$ in the $w$-model space $M_{w}^{n}$, i.e., it is the geodesic ball in $M_{w}^{n}$ such that $\operatorname{Vol}\left(D_{R}\right)=\operatorname{Vol}\left(B_{S(R)}^{w}\right)$. Equality in (2.3) for some fixed radius $R$ implies that $S(R)=R$ and $B_{R}^{n}$ and $B_{R}^{w}$ are isometric.

Outline of paper. We devote section 3 to the precise definitions of extrinsic balls and $w$-model comparison spaces, and to the description of the general setup of our comparison analysis. In sections 4, 5, and 6 we shall then prove Theorem 2.1, corollary 2.3, and corollary 2.4 respectively.

\section{Preliminaries}

We consider an immersed $m$-dimensional submanifold $P^{m}$ in a complete Riemannian manifold $N^{n}$. Let $p$ denote a point in $P$ and assume that $p$ is a pole of the ambient space $N$. We denote the distance function from $p$ in the ambient space by $r(x)=\operatorname{dist}_{N}(p, x)$ for all $x \in N$. Since $p$ is a pole there is - by definition - a unique geodesic from $p$ to $x$ which realizes the distance $r(x)$. We also denote by $r$ the restriction $\left.r\right|_{P}: P \longrightarrow \mathbb{R}_{+} \cup\{0\}$. This restriction is then called the extrinsic distance function from $p$ in $P^{m}$. The corresponding extrinsic metric balls of radius $R$ and center $p$ are denoted by $D_{R}(p) \subseteq P$ and defined as any precompact connected component (containing $p$ ) of the intersection

$$
B_{R}(p) \cap P=\{x \in P \mid r(x)<R\} \quad,
$$

where $B_{R}(p)$ denotes the geodesic $R$-ball around the pole $p$ in $N^{n}$. This intersection is always non-empty for $R>0$, and for sufficiently small $R$, the extrinsic ball $D_{R}(p)$ is also always precompact with a well-defined boundary $\partial D_{R}(p)$. 
When we consider the very special situation when $P^{m}$ is a totally geodesic submanifold $\mathbb{K}^{m}(b) \subseteq \mathbb{K}^{n}(b), n \geq m$, in the space form of constant curvature $b$, then the corresponding extrinsic $R$-ball $D_{R}^{b, m}(\tilde{p})$ centered at $\tilde{p} \in \mathbb{K}^{m}(b)$, is identical to a totally geodesic $R$-ball $B_{R}^{b, m}(\tilde{p})$ centered at $\tilde{p}$ in this submanifold, and the boundary $\partial B_{R}^{b, m}(\tilde{p})$ is simply the geodesic sphere $S_{R}^{b, m-1}(\tilde{p})$.

We now define our comparison spaces, the $w$-model spaces. We have used these spaces for similar comparison purposes in our previous work [MP3]. We refer to this paper, and also to the works [O'N], [Gri] and [GreW] for further details.

\subsection{The $w$-model spaces.}

Definition 3.1 (See [Gri], [GreW]). A $w$-model $M_{w}^{m}$ is a smooth warped product with base $B^{1}=[0, \Lambda[\subset \mathbb{R}$ (where $0<\Lambda \leq \infty)$, fiber $F^{m-1}=S_{1}^{m-1}$ (i.e. the unit $(m-1)$-sphere with standard metric), and warping function $w:\left[0, \Lambda\left[\rightarrow \mathbb{R}_{+} \cup\{0\}\right.\right.$ with $w(0)=0, w^{\prime}(0)=1$, and $w(r)>0$ for all $r>0$. The point $p_{w}=\pi^{-1}(0)$, where $\pi$ denotes the projection onto $B^{1}$, is called the center point of the model space. If $\Lambda=\infty$, then $p_{w}$ is a pole of $M_{w}^{m}$.

Remark 3.2. The simply connected space forms $\mathbb{K}^{m}(b)$ of constant curvature $b$ can be constructed as $w$-models with any given point as center point using the warping functions

$$
w(r)=Q_{b}(r)=\left\{\begin{array}{ll}
\frac{1}{\sqrt{b}} \sin (\sqrt{b} r) & \text { if } b>0 \\
r & \text { if } b=0 \\
\frac{1}{\sqrt{-b}} \sinh (\sqrt{-b} r) & \text { if } b<0
\end{array} .\right.
$$


Note that for $b>0$ the function $Q_{b}(r)$ admits a smooth extension to $r=\pi / \sqrt{b}$. For $b \leq 0$ any center point is a pole.

Proposition 3.3 (See [O'N] p. 206). Let $M_{w}^{m}$ be a $w$-model with warping function $w(r)$ and center $p_{w}$. The distance sphere of radius $r$ and center $p_{w}$ in $M_{w}^{m}$, denoted as $S_{r}^{w}$, is the fiber $\pi^{-1}(r)$. This distance sphere has the following constant mean curvature vector in $M_{w}^{m}$

$$
H_{\pi^{-1}(r)}=-\eta_{w}(r) \nabla^{M} \pi=-\eta_{w}(r) \nabla^{M} r
$$

where the mean curvature function $\eta_{w}(r)$ is defined by

$$
\eta_{w}(r)=\frac{w^{\prime}(r)}{w(r)}=\frac{d}{d r} \ln (w(r))
$$

In particular we have for the constant curvature space forms $\mathbb{K}^{m}(b)$ :

$$
\eta_{Q_{b}}(r)=\left\{\begin{array}{cl}
\sqrt{b} \cot (\sqrt{b} r) & \text { if } b>0 \\
1 / r & \text { if } b=0 \\
\sqrt{-b} \operatorname{coth}(\sqrt{-b} r) & \text { if } b<0
\end{array} .\right.
$$

Definition 3.4. Let $p$ be a point in a Riemannian manifold $M$ and let $x \in M-\{p\}$. The sectional curvature $K_{M}\left(\sigma_{x}\right)$ of the two-plane $\sigma_{x} \in T_{x} M$ is then called a $p$-radial sectional curvature of $M$ at $x$ if $\sigma_{x}$ contains the tangent vector to a minimal geodesic from $p$ to $x$. We denote these curvatures by $K_{p, M}\left(\sigma_{x}\right)$.

Proposition 3.5 (See [GreW] and [Gri]). Let $M_{w}^{m}$ be a $w$-model with center point $p_{w}$. Then the $p_{w}$-radial sectional curvatures of $M_{w}^{m}$ at every $x \in \pi^{-1}(r)$ (for $r>0$ ) are all identical and determined by the 
radial function $K_{w}(r)$ defined as follows:

$$
K_{p_{w}, M_{w}}\left(\sigma_{x}\right)=K_{w}(r)=-\frac{w^{\prime \prime}(r)}{w(r)} .
$$

For any given warping function $w(r)$ we introduce the isoperimetric quotient function $q_{w}(r)$ for the corresponding $w$-model space $M_{w}^{m}$ as follows:

$$
q_{w}(r)=\frac{\operatorname{Vol}\left(B_{r}^{w}\right)}{\operatorname{Vol}\left(S_{r}^{w}\right)}=\frac{\int_{0}^{r} w^{m-1}(t) d t}{w^{m-1}(r)},
$$

where $B_{r}^{w}$ denotes the polar centered geodesic $r$-ball of radius $r$ in $M_{w}^{m}$ with boundary sphere $S_{r}^{w}$.

Since the warping function $w$ usually appears raised to the power $m-1$ we will use the notion $a_{w}(r)$ to denote this power of $w$ :

$$
\begin{aligned}
& a_{w}(r)=\operatorname{Vol}\left(S_{r}^{w}\right) / V_{0}=w^{m-1}(r), \\
& q_{w}(r)=\frac{\int_{0}^{r} a_{w}(t) d t}{a_{w}(r)} .
\end{aligned}
$$

Note that although $q_{w}(r)$ and $a_{w}(r)$ depend on the dimension $m$ we suppress this dependence from the notations, since in each case the dimension will be evident.

Then we have the following observations concerning the mean exit time function and the torsional rigidity of $B_{r}^{w}$ in terms of $q_{w}$ and $a_{w}$ :

\section{Proposition 3.6.}

$$
E_{R}^{w}(r)=\int_{r}^{R} q_{w}(t) d t
$$

$$
\mathcal{A}_{1}\left(B_{R}^{w}\right)=\int_{B_{R}^{w}} E_{R}^{w} d \mu=V_{0} \int_{0}^{R} a_{w}(r)\left(\int_{r}^{R} q_{w}(t) d t\right) d r
$$


Differentiating with respect to $R$ gives

$$
\begin{aligned}
\frac{d}{d R} \mathcal{A}_{1}\left(B_{R}^{w}\right) & =V_{0} q_{w}(R) \int_{0}^{R} a_{w}(r) d r \\
& =\frac{\operatorname{Vol}\left(B_{R}^{w}\right)^{2}}{\operatorname{Vol}\left(S_{R}^{w}\right)} \\
& =q_{w}^{2}(R) \operatorname{Vol}\left(S_{R}^{w}\right) .
\end{aligned}
$$

Upon integration of the latter equality we get the following alternative expression for the torsional rigidity of the geodesic balls in the w-model spaces, which may be of independent interest:

$$
\mathcal{A}_{1}\left(B_{R}^{w}\right)=\int_{B_{R}^{w}} q_{w}^{2} d \sigma
$$

Remark 3.7. Since $q_{w}(r)>0$, it follows from (3.8) that for fixed $r$, the mean exit time function $E_{R}^{w}(r)$ is an increasing function of $R$. Furthermore if $q_{w}^{\prime}(r) \geq 0$ then the average mean exit time $\mathcal{A}_{1}\left(B_{r}^{w}\right) / \operatorname{Vol}\left(B_{r}^{w}\right)$ of $B_{r}^{w}$ is also a non-decreasing function of $r$. This relates to the question of finding $w$-model spaces with bounded average mean exit time at infinity. We will return to this question in section 5 .

Concerning $q_{w}^{\prime}(r) \geq 0$ we now display alternative ways to express this growth condition for the isoperimetric quotient:

Observation 3.8. The following conditions on the warping function $w(r)$ are equivalent (recall that $\eta_{w}(r)$ denotes the mean curvature of the fiber at $r$ of the model space $\left.M_{w}^{m}\right)$ :

$$
\begin{aligned}
q_{w}^{\prime}(r) & \geq 0 \\
q_{w}(r) \eta_{w}(r) & \leq 1 /(m-1) \\
w^{m}(r) & \geq(m-1) w^{\prime}(r) \int_{0}^{r} w^{m-1}(t) d t .
\end{aligned}
$$


Definition 3.9. A given $w$-model space satisfying one and hence all three of these conditions will be called balanced from above. The balancing condition is thus guaranteed by the product of the isoperimetric quotient and the mean curvature being bounded from above by $1 /(m-1)$.

Observation 3.10. The following conditions on the warping function $w(r)$ of a model space are also equivalent

$$
\begin{aligned}
q_{w}(r) \eta_{w}(r) & \geq 1 / m \\
w^{m}(r) & \leq m w^{\prime}(r) \int_{0}^{r} w^{m-1}(t) d t .
\end{aligned}
$$

Definition 3.11. A given $w$-model space satisfying one, hence both, of the conditions in observation 3.10 will be called balanced from below. If $M_{w}^{m}$ is balanced from above and from below, it will simply be called totally balanced.

The following are also immediate consequences of the definitions:

Observation 3.12. If $K_{w}(r) \geq-\eta_{w}^{2}(r)$ then $M_{w}^{m}$ is balanced from above. If $K_{w}(r) \leq 0$ then $M_{w}^{m}$ is balanced from below.

In this sense, then, there are many $w$-model spaces which are totally balanced.

Examples 3.13. The following are typical examples of the four types of balancing and non-balancing of $w$-model spaces (the proofs are straightforward):

(1) Every positively curved space form of constant positive curvature is (within its injectivity radius) balanced from above but it is not balanced from below. 
(2) Every space form model space of constant non-positive curvature is totally balanced.

(3) The warping function $w(r)=\log (1+r)$ gives a $w$-model space which is balanced from above but not from below.

(4) The function $w(r)=\exp \left(r^{2}\right)+r-1$ gives a $w$-model space which is balanced from below but not from above. Recall that $w(r)$ must satisfy the initial conditions $w(0)=0$ and $w^{\prime}(0)=$ 1 in order to generate a model space with a well-defined pole.

(5) Finally, suitable combinations of 'shifted' versions of the examples $w(r)$ from examples (2) and (3) will give model spaces which are balanced neither from above nor from below.

3.2. Symmetrization into model spaces. Another instrumental concept needed to prove our result is a generalization of the concept of Schwarz symmetrization in $\mathbb{R}^{n}$. We extend the symmetrization to allow suitable $w$-model spaces $M_{w}^{m}$ as target spaces.

Definition 3.14. Suppose $D$ is a precompact open connected domain in $P^{m}$. Then the $w$-model space symmetrization of $D$ is denoted by $D^{*}$ and is defined to be the unique $p_{w}$-centered ball $D^{*}=B^{w}(D)$ in $M_{w}^{m}$ satisfying $\operatorname{Vol}(D)=\operatorname{Vol}\left(B^{w}(D)\right)$. In the particular case where $D$ is actually an extrinsic metric ball $D_{R}$ in $P$ of radius $R$ we may write

$$
D_{R}^{*}=B^{w}(D)=B_{S(R)}^{w}
$$

where $S(R)$ is some increasing function of $R$ which depends on the geometry of $P$, according to the defining property:

$$
\operatorname{Vol}\left(D_{R}\right)=\operatorname{Vol}\left(B_{S(R)}^{w}\right)
$$


Remark 3.15. Since $w$-model spaces may have finite volume, there may not in general be enough room for such symmetrization constructions. However, for $w$-model spaces balanced from below the volume $\operatorname{Vol}\left(B_{r}^{w}\right)$ increases to infinity with $r$ because the assumption $\eta_{w}(r)>0$ implies that $w^{\prime}(r)>0$ as well.

We also introduce the notion of a symmetrized function on the symmetrization $D^{*}$ of $D$ as follows.

Definition 3.16. Let $f$ denote a nonnegative function on $D$

$$
f: D \subseteq P \rightarrow \mathbb{R} \cup\{0\}
$$

For $t>0$ we let

$$
D(t)=\{x \in D \mid f(x) \geq t\} \quad .
$$

Then the symmetrization of $f$ is the function $f^{*}: D^{*} \rightarrow \mathbb{R} \cup\{0\}$ defined by

$$
f^{*}\left(x^{*}\right)=\sup \left\{t \mid x^{*} \in D(t)^{*}\right\}
$$

Proposition 3.17. The symmetrized objects $f^{*}$ and $D^{*}$ satisfy the following properties:

(1) The function $f^{*}$ depends only on the geodesic distance to the center $p_{w}$ of the ball $D^{*}$ in $M_{w}^{m}$ and is non-increasing.

(2) The functions $f$ and $f^{*}$ are equimeasurable in the sense that

$$
\operatorname{Vol}_{P}(\{x \in D \mid f(x) \geq t\})=\operatorname{Vol}_{M_{w}^{m}}\left(\left\{x^{*} \in D^{*} \mid f^{*}\left(x^{*}\right) \geq t\right\}\right)
$$

for all $t \geq 0$. In particular, for all $t>0$, we have

$$
\int_{D(t)} f d \mu \leq \int_{D(t)^{*}} f^{*} d \sigma
$$


Remark 3.18. The proof of these properties follows the proof of the classical Schwarz symmetrization using the 'slicing' technique for symmetrized volume integrations and comparison - see e.g. [Ch2].

The proof of Theorem 2.1 is based mainly upon two results, Theorem A and Theorem B, concerning the mean exit time function defined on the extrinsic balls and an isoperimetric property satisfied by these extrinsic domains. These theorems were established for extrinsic balls in minimal submanifolds of a Riemannian manifold with bounded geometry, namely, with an upper bound on its sectional curvatures, (in particular, the ambient space in Theorem B was assumed to be an Hadamard-Cartan manifold). However, we can use the Hessian and Laplacian comparison theory based on Theorem 3.19, to conclude a comparison, corollary 3.20, for the Laplacian of radial functions defined on the extrinsic balls of the submanifold $P^{m}$ in a manifold with a pole $N^{n}$ satisfying our hypotheses, (see [MP3] for more details).

Theorem 3.19 (See [GreW], Theorem A). Let $N^{n}$ be a manifold with a pole $p$, let $M_{w}^{m}$ denote a $w$-model with center $p_{w}$. Suppose that $m \leq n$ and that every $p$-radial sectional curvature at $x \in N-\{p\}$ is bounded from above by the $p_{w}$-radial sectional curvatures in $M_{w}^{m}$ as follows:

$$
K_{p, N}\left(\sigma_{x}\right) \leq-\frac{w^{\prime \prime}(r)}{w(r)}
$$


for every radial two-plane $\sigma_{x} \in T_{x} N$ at distance $r=r(x)=\operatorname{dist}_{N}(p, x)$ from $p$ in $N$. Then the Hessian of the distance function in $N$ satisfies

$$
\begin{aligned}
\operatorname{Hess}^{N}(r(x))(X, X) & \geq \operatorname{Hess}^{M_{w}^{m}}(r(y))(Y, Y) \\
& =\eta_{w}(r)\left(1-\left\langle\nabla^{M} r(y), Y\right\rangle_{M}^{2}\right) \\
& =\eta_{w}(r)\left(1-\left\langle\nabla^{N} r(x), X\right\rangle_{N}^{2}\right)
\end{aligned}
$$

for every unit vector $X$ in $T_{x} N$ and for every unit vector $Y$ in $T_{y} M$ with $r(y)=r(x)=r$ and $\left\langle\nabla^{M} r(y), Y\right\rangle_{M}=\left\langle\nabla^{N} r(x), X\right\rangle_{N}$.

Corollary 3.20. Suppose again that the assumptions of Theorem 3.19 are satisfied. Then we have for every smooth function $f(r)$ with $f^{\prime}(r) \geq$ 0 for all $r$, (respectively $f^{\prime}(r) \leq 0$ for all $\left.r\right)$ :

$$
\begin{aligned}
\Delta^{P}(f \circ r) \geq(\leq) & \left(f^{\prime \prime}(r)-f^{\prime}(r) \eta_{w}(r)\right)\left\|\nabla^{P} r\right\|^{2} \\
& +m f^{\prime}(r)\left(\eta_{w}(r)+\left\langle\nabla^{N} r, H_{P}\right\rangle\right)
\end{aligned}
$$

where $H_{P}$ denotes the mean curvature vector of $P$ in $N$.

Using corollary 3.20 along the lines of the proofs in [Ma1] and [Pa], we obtain the generalized statements below. These theorems and their proofs concern extrinsic balls in minimal submanifolds of a manifold with a pole, but can also be stated and proved for codimension-0 extrinsic balls in the ambient manifold, i.e. geodesic balls in $N$, a setting in which the balancing condition from below (for the $w$-model spaces) is then not needed. In order to gain clarity and completeness we state and prove the theorems concerning extrinsic balls in minimal submanifolds in this section, and give an explicit sketch of the proof in the case of intrinsic geodesic balls in section 6. These latter comparison results are explicitly used in the proof of corollary 2.4 . 
Theorem A ([Ma1]). Let $\left\{N^{n}, P^{m}, M_{w}^{m}\right\}$ denote a comparison constellation, and assume that $M_{w}^{m}$ is balanced from below. Let $D_{R}$ be an extrinsic $R$-ball in $P^{m}$, with center at a point $p \in P$ which also serves as a pole for the ambient space $N$. Then

$$
E_{R}(x) \leq E_{R}^{w}(x) \text { for all } x \in D_{R}
$$

where $E_{R}^{w}$ denotes the mean exit time function defined on the geodesic $R$-ball in the $w$-model space $M_{w}^{m}$. Moreover, if equality in (3.19) is fulfilled on $D_{R}$ and the balance of $M_{w}^{m}$ from below is sharp $q_{w}(r) \eta_{w}(r)>$ $1 / m$ for all $r$, then $D_{R}$ is a minimal cone in the ambient space $N^{n}$, so if $N^{n}$ is actually the hyperbolic space $\mathbb{K}^{n}(b)$, then $P^{m}$ is totally geodesic in $\mathbb{K}^{n}(b)$.

Remark 3.21. Concerning the balance condition, we observe that $w(r)$ satisfies $q_{w}(r) \eta_{w}(r)=1 / m$ for all $r$ (with the standard initial conditions) if and only if $w(r)=r$. The $w$-model space $M_{w}^{m}$ is then the flat Euclidean space $\mathbb{R}^{m}$ in which every minimal submanifold is known to have the same mean exit time function $E_{R}(x)=\left(R^{2}-r^{2}(x)\right) / 2 m$ for all extrinsic balls $D_{R}$, see [Ma1]. In this case, therefore, we do not obtain further structural information from equality in equation (3.19).

Proof. The proof we are going to sketch follows the lines of [Ma1], where the result was established for ambient manifolds with bounded sectional curvatures from above. Let us consider $E_{R}^{w}(r)$ the (radial) solution of the Poisson equation with Dirichlet boundary data defined on the geodesic $R$-ball $B_{R}^{w}$ in the $w$-model space $M_{w}^{m}$,

$$
\begin{aligned}
\Delta^{M_{w}^{m}} E & =-1 \text { on } B_{R}^{w} \\
\left.E\right|_{S_{R}^{w}} & =0
\end{aligned}
$$


In order to compare this solution $E_{R}(x)$ with the solution of the corresponding Poisson problem defined in $D_{R} \subseteq P$, we transplant $E_{R}^{w}(r)$ to $D_{R}$ :

$$
E_{R}^{w}: D_{R} \longrightarrow \mathbb{R} ; \quad E_{R}^{w}(x):=E_{R}^{w}(r(x))
$$

Since $E_{R}^{w^{\prime}}(r) \leq 0$ for all $r \leq R$, we may apply corollary 3.20 to obtain

$$
\begin{aligned}
\Delta^{P} E_{R}^{w} & \leq\left(E^{w^{\prime \prime}}(r)-E^{w^{\prime}}(r) \eta_{w}(r)\right)\left\|\nabla^{P} r\right\|^{2} \\
& +m E^{w^{\prime}}(r)\left(\eta_{w}(r)+\left\langle\nabla^{N} r, H_{P}\right\rangle\right)
\end{aligned}
$$

Then, as $w^{m}(r) \leq m w^{\prime}(r) \int_{0}^{r} w^{m-1}(t) d t$, it is easy to check that $E^{w^{\prime \prime}}(r)-$ $E^{w^{\prime}}(r) \eta_{w}(r) \geq 0$, and taking into account that $\left\|\nabla^{P} r\right\|^{2} \leq 1$ and that $P$ is minimal, (3.21) becomes

$$
\begin{aligned}
\Delta^{P} E_{R}^{w} & \leq E^{w^{\prime \prime}}(r)+(m-1) E^{w^{\prime}}(r) \eta_{w}(r) \\
& =\Delta^{M_{w}^{m}} E_{R}^{w}=-1=\Delta^{P} E_{R}
\end{aligned}
$$

Hence, as $\left.E_{R}^{w}\right|_{\partial D_{R}}=\left.E_{R}\right|_{\partial D_{R}}=0$, we have $E_{R} \leq E_{R}^{w}$ on $D_{R}$ by applying the Maximum Principle.

If we have equality in (3.19) for some fixed radius $R$, inequalities in (3.22) and (3.21) become equalities. Therefore, $\left\|\nabla^{P} r\right\|=1=\left\|\nabla^{N} r\right\|$ in $D_{R}$, so $\nabla^{P} r=\nabla^{N} r$ on $D_{R}$. All the geodesics in $N$ starting at $p$ thus lies in $P$, so $D_{R}=\exp _{p}\left(\tilde{D}_{R}\right)$, being $\tilde{D}_{R}$ the 0 -centered $R$-ball in $T_{p} P$. Hence, $D_{R}$ is a minimal cone in $N$, in fact, the geodesic $R$-ball $B_{R}^{m}$ in $P$. If $N=\mathbb{K}^{n}(b),(b<0)$, the hyperbolic space with constant curvature $b$, then, from analytical continuation from $D_{R}=B_{R}^{m}$, we finally get that $P^{m}$ is a totally geodesic submanifold of $\mathbb{K}^{n}(b)$. 
The second theorem is based on the theorem above and describes an isoperimetric inequality satisfied by the extrinsic balls in the same setting.

Theorem B $([\mathrm{Pa}])$. Let $\left\{N^{n}, P^{m}, M_{w}^{m}\right\}$ denote a comparison constellation, and assume that $M_{w}^{m}$ is balanced from below. Let $D_{R}$ be an extrinsic $R$-ball in $P^{m}$, with center at a point $p \in P$ which is also a pole in the ambient space $N$. Then

$$
\frac{\operatorname{Vol}\left(\partial D_{R}\right)}{\operatorname{Vol}\left(D_{R}\right)} \geq \frac{\operatorname{Vol}\left(S_{R}^{w}\right)}{\operatorname{Vol}\left(B_{R}^{w}\right)} \quad \text { for all } R>0
$$

Furthermore,

$$
\operatorname{Vol}\left(D_{R}\right) \geq \operatorname{Vol}\left(B_{R}^{w}\right) \text { for all } R>0
$$

Moreover, if equality in inequalities (3.23) or (3.24) holds for some fixed radius $R$ and if again the balance of $M_{w}^{m}$ from below is sharp $q_{w}(r) \eta_{w}(r)>1 / m$ for all $r$, then $D_{R}$ is a minimal cone in the ambient space $N^{n}$, so if $N^{n}$ is the hyperbolic space $\mathbb{K}^{n}(b), b<0$, then $P^{m}$ is totally geodesic in $\mathbb{K}^{n}(b)$.

Proof. The proof of this result is based on Theorem A and an application of the divergence theorem and the co-area formula. It follows the lines of $[\mathrm{Pa}]$, where the result is proved in cases where the ambient space is an Hadamard-Cartan manifold.

In the model spaces $M_{w}^{m}$, using the Poisson equation $\Delta E_{R}^{w}=-1$ on $B_{R}^{w}$, the divergence theorem, and the fact that $\frac{\nabla^{P} r}{\left\|\nabla^{P} r\right\|}$ is the unit normal pointing outward from the boundary $\partial D_{R}$, we have, (in this context 
where $D_{R}=B_{R}^{w}$ and $\partial B_{R}^{w}=S_{R}^{w}$,

$$
\begin{aligned}
\operatorname{Vol}\left(B_{R}^{w}\right) & =-\int_{B_{R}^{w}} \Delta^{M_{w}^{m}} E_{R}^{w} d \mu \\
& =\int_{S_{R}^{w}}<\nabla E_{R}^{w}, \nabla^{M_{w}^{m}} r>d \sigma=E_{R}^{w^{\prime}}(R) \operatorname{Vol}\left(S_{R}^{w}\right) .
\end{aligned}
$$

Hence,

$$
E_{R}^{w^{\prime}}(R)=-\frac{\operatorname{Vol}\left(B_{R}^{w}\right)}{\operatorname{Vol}\left(S_{R}^{w}\right)} \quad \text { for all } R \geq 0
$$

Now, we proceed as in the proof of Theorem A: by transplantation of $E_{R}^{w}$ to $D_{R}$, we get inequality $\Delta^{P} E_{R}^{w} \leq-1$, so, applying the divergence theorem, taking the unit normal to $\partial D_{R}$ as $\frac{\nabla^{P} r}{\left\|\nabla^{P} r\right\|}$, and using $\left\|\nabla^{P} r\right\| \leq$ 1 , we get

$$
\begin{aligned}
\operatorname{Vol}\left(D_{R}\right) & =\int_{D_{R}}-\Delta^{P} E_{R} d \mu \\
& \leq \int_{D_{R}}-\Delta^{P} E_{R}^{w} d \mu \\
& =-\int_{D_{R}} \operatorname{div}\left(\nabla^{P} E_{R}^{w}\right) d \mu \\
& =-\int_{\partial D_{R}}<\nabla^{P} E_{R}^{w}, \frac{\nabla^{P} r}{\left\|\nabla^{P} r\right\|}>d \mu \\
& =-E_{R}^{w^{\prime}}(R) \int_{\partial D_{R}}\left\|\nabla^{P} r\right\| d \mu \\
& \leq \frac{\operatorname{Vol}\left(B_{R}^{w}\right)}{\operatorname{Vol}\left(S_{R}^{w}\right)} \operatorname{Vol}\left(\partial D_{R}\right),
\end{aligned}
$$

which shows inequality (3.23).

Equality in (3.23) for some fixed radius $R$ implies that inequalities in (3.27) become equalities, so we obtain

$$
\int_{D_{R}}-\Delta^{P} E_{R} d \mu=\int_{D_{R}}-\Delta^{P} E_{R}^{w} d \mu
$$


which, together with inequality (3.22), implies that $\Delta^{P} E_{R}=\Delta^{P} E_{R}^{w}$ in $D_{R}$, which in turn implies that $\left\|\nabla^{P} r\right\|=1=\left\|\nabla^{N} r\right\|$ in $D_{R}$, so $D_{R}$ is a minimal cone in $N$, in fact, the geodesic $R$-ball $B_{R}^{m}$ in $P$, in the same way as in Theorem A.

The proof of inequality (3.24) from inequality (3.23) is based on the co-area formula, and follows as before the lines in $[\mathrm{Pa}]$ : Apply the co-area formula to the level sets constructed by means of the smooth function $h: D_{R} \longrightarrow \mathbb{R}$, defined as $h(q):=R^{2}-r^{2}(q)$ for all $q \in D_{R}$, where $r(q)$ is the extrinsic distance from $q$ to the pole $p$. We conclude that $\frac{d}{d r} \operatorname{Vol}\left(D_{r}\right) \geq \operatorname{Vol}\left(\partial D_{r}\right)$.

Now consider the function

$$
G(r)= \begin{cases}\ln \left(\frac{\operatorname{Vol}\left(D_{r}\right)}{\operatorname{Vol}\left(B_{r}^{w}\right)}\right) & \text { if } r>0 \\ 0 & \text { if } r=0\end{cases}
$$

It is straightforward to check that $G(r)$ is continuous using the asymptotic expansion for the volume of extrinsic balls with small radii in a submanifold of an arbitrary Riemannian manifold established in [KP] and the identity

$$
\operatorname{Vol}\left(B_{R}^{w}\right)=\int_{0}^{R} \operatorname{Vol}\left(S_{r}^{w}\right) d r=\operatorname{Vol}\left(S_{1}^{0, m-1}\right) \int_{0}^{R} w^{m-1}(r) d r
$$

In view of (3.29) it is straightforward to check, using inequality (3.23), that $G^{\prime}(r) \geq 0$ for all $r \leq R$, so $G(r) \geq 0$ for all $r \leq R$. In the same way as before, equality in (3.24) implies that $\left\|\nabla^{P} r\right\|=$ $1=\left\|\nabla^{N} r\right\|$ in $D_{R}$ 
As mentioned before, these results will allow us to avoid the main condition in $[\mathrm{Mc}]$ referring to an isoperimetric condition for all domains in comparison with constant curvature space forms.

\section{Proof of Theorem 2.1}

Given the solutions $E_{R}$ and $E_{R}^{w}$ to the Poisson equation with Dirichlet boundary data defined on $D_{R}$ and $B_{R}^{w}$ respectively, we compare them by transplanting $E_{R}^{w}$ to $D_{R}$ as before.

Using Theorem A and the property (3.15) of the symmetric model space rearrangement of the extrinsic ball $D_{R}$, we have that

$$
\begin{aligned}
\mathcal{A}_{1}\left(D_{R}\right) & =\int_{D_{R}} E_{R} d \mu \\
& \leq \int_{D_{R}} E_{R}^{w} d \mu \\
& \leq \int_{D_{R}^{*}} E_{R}^{w *} d \sigma \\
& =\int_{B_{S(R)}^{w}} E_{R}^{w *} d \sigma
\end{aligned}
$$

where the symmetrization $D_{R}^{*}$ of $D_{R}$ is the geodesic $S(R)$-ball in $M_{w}^{m}$, $B_{S(R)}^{w}$, satisfying $\operatorname{Vol}\left(D_{R}\right)=\operatorname{Vol}\left(B_{S(R)}^{w}\right)$. From inequality (3.24) we have that $S(R) \geq R$.

The symmetrized function $E_{R}^{w *}$ is a radial function defined on $B_{S(R)}^{w}$ but it does not necessarily satisfy the Poisson equation there. We therefore consider the radial solution $E_{S(R)}^{w}$ to the Poisson equation with Dirichlet boundary data defined on $B_{S(R)}^{w}$,

$$
\begin{aligned}
\Delta^{M_{w}^{m}} E & =-1 \\
\left.E\right|_{\partial B_{S(R)}^{w}} & =0
\end{aligned}
$$


The idea is to compare this Poisson solution with the symmetrized function $E_{R}^{w *}$ in order to prove the following

\section{Proposition 4.1.}

$$
E_{R}^{w *^{\prime}}(r) \geq E_{S(R)}^{w^{\prime}}(r) \text { for all } r \in[0, S(R)]
$$

Integrating both sides of inequality (4.3) with respect to $r$, from $r=t$ to $r=S(R)$, and taking into account that $E_{R}^{w *}(S(R))=$ $E_{S(R)}^{w}(S(R))=0$, we finally obtain

$$
E_{R}^{w *}(t) \leq E_{S(R)}^{w}(t) \text { for all } t \in[0, S(R)],
$$

so that with the inequality from (4.1) we finally have:

$$
\begin{aligned}
\mathcal{A}_{1}\left(D_{R}\right) & \leq \int_{B_{S(R)}^{w}} E_{R}^{w *} d \sigma \\
& \leq \int_{B_{S(R)}^{w}} E_{S(R)}^{w}=\mathcal{A}_{1}\left(B_{S(R)}^{w}\right) d \sigma,
\end{aligned}
$$

which proves the inequality (2.1) in theorem 2.1. We now return to the

Proof of Proposition 4.1. To prove inequality (4.3), let us consider $T=$ $\max _{B_{R}^{w}} E_{R}^{w}$. On the other hand, and given $t \in[0, T]$, let us define the sets

$$
D(t)=\left\{x \in D_{R} \mid E_{R}^{w}(r(x)) \geq t\right\} \quad,
$$

and

$$
\Gamma(t)=\left\{x \in D_{R} \mid E_{R}^{w}(r(x))=t\right\} \quad .
$$

Since $E_{R}^{w}(r)$ is radial and nonincreasing, (see [Ma1]), the maximum $T$ will be attained at $r=0, D(t)$ is the extrinsic ball in $P$ with radius $g(t):=E_{R}^{w^{-1}}(t)$, that we denote as $D_{g(t)}$, and $\Gamma(t)$ is its boundary, the 
extrinsic sphere with radius $g(t), \partial D_{g(t)}$. We have too that $D(0)=D_{R}$ and $D(T)=\{p\}$, the center of the extrinsic ball $D_{R}$.

We consider the symmetrizations of the sets $D(t) \subseteq P$, namely, the geodesic balls $D(t)^{*}=B_{r(t)}^{w}$ in $M_{w}^{m}$ such that

$$
\operatorname{Vol}(D(t))=\operatorname{Vol}\left(D_{g(t)}\right)=\operatorname{Vol}\left(B_{r(t)}^{w}\right)
$$

As before, we have from Theorem B (using here the condition, that the $w$-model space is balanced from below) that $r(t) \geq g(t)$ for all $t \in$ $[0, T]$. We also consider the geodesic spheres $S_{r(t)}^{w}$, which are the boundaries of these symmetrizations, i.e. $\partial D(t)^{*}=S_{r(t)}^{w}$.

Hence, we have defined a nonincreasing function

$$
r:[0, T] \longrightarrow[0, S(R)] ; r=r(t)
$$

with inverse

$$
\psi:[0, S(R)] \longrightarrow[0, T] ; \psi=\psi(r)
$$

such that $\psi^{\prime}(r)=\frac{1}{r^{\prime}(t)}<0$.

Now, we also have that $E_{R}^{w *^{\prime}}(r)=\psi^{\prime}(r)=\frac{1}{r^{\prime}(t)}$ for all $t \in[0, T]$. To see this we must take into account that $B_{S(R)}^{w}=\cup_{t \in[0, T]} \partial D(t)^{*}$, so, given $x \in B_{S(R)}^{w}$, there exists some biggest value $t_{0}$ such that $r(x)=r\left(t_{0}\right)$, (and hence, $\left.x \in D\left(t_{0}\right)^{*}\right)$, so, by definition of the symmetric function, $E_{R}^{w *}(x)=E_{R}^{w *}(r(x))=t_{0}=\psi(r(x))$, and therefore,

$$
E_{R}^{w *^{\prime}}(r(t))=\frac{1}{r^{\prime}(t)} \text { for all } t \in[0, T]
$$

We now need the following 


\section{Lemma 4.2.}

$$
r^{\prime}(t)=\frac{-1}{\operatorname{Vol}\left(S_{r(t)}^{w}\right)} \int_{\Gamma(t)}\left\|\nabla^{P} E_{R}^{w}\right\|^{-1} d \mu
$$

Proof. Let us denote by $W(r)$ the volume of the geodesic $r$-ball in $M_{w}^{m}$, namely, $W(r):=\operatorname{Vol}\left(B_{r}^{w}\right)$. Then, we can define $V:[0, T] \longrightarrow \mathbb{R}$ as $V(t):=\operatorname{Vol}\left(B_{r(t)}^{w}\right)=W(r(t))$, so, by the chain rule and the fact that $W^{\prime}(r)=\operatorname{Vol}\left(S_{r}^{w}\right)$, we have

$$
V^{\prime}(t)=\operatorname{Vol}\left(S_{r}^{w}\right) r^{\prime}(t)
$$

As an application of the co-area formula (as given in [Ch1]) to the level sets $\Gamma(t)$, defined by the function $E_{R}^{w}$, we then obtain the claimed identity:

$$
\operatorname{Vol}\left(S_{r}^{w}(t)\right) r^{\prime}(t)=-\int_{\Gamma(t)}\left\|\nabla^{P} E_{R}^{w}\right\|^{-1} d \mu
$$

Since $E_{R}^{w}$ is radial, and $\Gamma(t)=\partial D_{g(t)}$, we get, taking into account that $\left\|\nabla^{P} r\right\| \leq 1$ and that, (see $[\mathrm{Pa}]$ ),

$$
E_{R}^{w^{\prime}}(r)=-\frac{\operatorname{Vol}\left(B_{r}^{w}\right)}{\operatorname{Vol}\left(S_{r}^{w}\right)}
$$

that also

$$
\begin{gathered}
\int_{\Gamma(t)}\left\|\nabla^{P} E_{R}^{w}\right\|^{-1} d \mu=\left|E_{R}^{w^{\prime}}(g(t))\right|^{-1} \int_{\partial D_{g(t)}}\left\|\nabla^{P} r\right\|^{-1} d \mu \\
\geq \frac{\operatorname{Vol}\left(S_{g(t)}^{w}\right)}{\operatorname{Vol}\left(B_{g(t)}^{w}\right)} \operatorname{Vol}\left(\partial D_{g(t)}\right)
\end{gathered}
$$

But the function $\frac{\operatorname{Vol}\left(S_{r}^{w}\right)}{\operatorname{Vol}\left(B_{r}^{w}\right)}$ is non-increasing for all values of $r$ by the assumption that the model space is balanced from above. Indeed, the quotient is $1 / q_{w}(r)$ which is non-increasing since $q_{w}^{\prime}(r)>0$. Then, as 
$r(t) \geq g(t)$, we have, using (3.10),

$$
-\int_{\Gamma(t)}\left\|\nabla^{P} E_{R}^{w}\right\|^{-1} d \mu \leq-\frac{\operatorname{Vol}\left(S_{r(t)}^{w}\right)}{\operatorname{Vol}\left(B_{r(t)}^{w}\right)} \operatorname{Vol}\left(\partial D_{g(t)}\right)
$$

and, hence,

$$
\frac{1}{r^{\prime}(t)} \geq-\frac{\operatorname{Vol}\left(B_{r(t)}^{w}\right)}{\operatorname{Vol}\left(\partial D_{g(t)}\right)}
$$

$\operatorname{But} \operatorname{Vol}\left(B_{r(t)}^{w}\right)=\operatorname{Vol}\left(D_{g(t)}\right)$, so using the isoperimetric inequality satisfied by the extrinsic balls in minimal submanifolds stated in Theorem B and the fact that $\frac{\operatorname{Vol}\left(S_{r}^{w}\right)}{\operatorname{Vol}\left(B_{r}^{w}\right)}$ is non-increasing, we finally obtain,

$$
\begin{aligned}
\frac{1}{r^{\prime}(t)} & \geq-\frac{\operatorname{Vol}\left(D_{g(t)}\right)}{\operatorname{Vol}\left(\partial D_{g(t)}\right)} \geq-\frac{\operatorname{Vol}\left(B_{g(t)}^{w}\right)}{\operatorname{Vol}\left(S_{g(t)}^{w}\right)} \\
& \geq-\frac{\operatorname{Vol}\left(B_{r(t)}^{w}\right)}{\operatorname{Vol}\left(S_{r(t)}^{w}\right)}=E_{S(R)}^{w^{\prime}}(r(t))
\end{aligned}
$$

and hence the statement in Proposition 4.1

$$
-E_{R}^{w *^{\prime}}(r(t))=-\frac{1}{r^{\prime}(t)} \leq-E_{S(R)}^{w^{\prime}}(r(t)) \text { for all } t \in[0, T]
$$

We now discuss the equality assertion in Theorem 2.1. If we have equality in (2.1), for some fixed radius $R_{0}$, then inequalities in (4.1) become equalities for this fixed radius, so we have that

$$
E_{R}=E_{R}^{w} \text { on } D_{R_{0}}
$$

When the balance of the model space is strict, and by virtue of the equality assertion in the statement of Theorem $\mathrm{A}$, this implies that $D_{R_{0}}$ is a minimal cone in the submanifold $P^{m}$. If the ambient space $N^{n}$ is 
the hyperbolic space, then $P^{m}$ is totally geodesic in $N=\mathbb{K}^{n}(b)$, (see [Ma1]). This finishes the proof of Theorem 2.1.

\section{Proof of COROLlary 2.3}

Concerning the behavior of the average mean exit time at infinity for model spaces, we have the following first result which we need to prove the corollary:

Proposition 5.1. Let $M_{w}^{m}$ denote a $w$-model space. Then the average mean exit time from the $R$-balls in the space is controlled by the isoperimetric function $q_{w}(r)$ as follows. Suppose that

$$
\lim _{R \rightarrow \infty} q_{w}(R)=q_{w}(\infty)<\infty
$$

Then

$$
\lim _{R \rightarrow \infty}\left(\frac{\mathcal{A}_{1}\left(B_{R}^{w}\right)}{\operatorname{Vol}\left(B_{R}^{w}\right)}\right)=q_{w}^{2}(\infty)
$$

Proof. This follows directly from equation (3.10) together with an application of L'Hospitals rule as follows:

$$
\begin{aligned}
\lim _{R \rightarrow \infty}\left(\frac{\frac{d}{d R} \mathcal{A}_{1}\left(B_{R}^{w}\right)}{\frac{d}{d R} \operatorname{Vol}\left(B_{R}^{w}\right)}\right) & =\lim _{R \rightarrow \infty}\left(\frac{q_{w}^{2}(R) \operatorname{Vol}\left(S_{R}^{w}\right)}{\operatorname{Vol}\left(S_{R}^{w}\right)}\right) \\
& =\lim _{R \rightarrow \infty}\left(q_{w}^{2}(R)\right) \\
& =\left(\lim _{R \rightarrow \infty} q_{w}(R)\right)^{2}
\end{aligned}
$$

Corollary 5.2. Let $w(r)=Q_{b}(r)$ for $b \leq 0$. Then the average mean exit time from the $R$-balls in the corresponding $m$-dimensional space 
form model of constant curvature b satisfies:

$$
\lim _{R \rightarrow \infty}\left(\frac{\mathcal{A}_{1}\left(B_{R}^{w}\right)}{\operatorname{Vol}\left(B_{R}^{w}\right)}\right)=\left\{\begin{array}{l}
\infty \quad \text { for } b=0 \\
(m-1)^{-2} b^{-2} \text { for } b<0
\end{array} .\right.
$$

From this we observe in particular, that 'totally balanced' does not imply that $q_{w}(\infty)<\infty$, since the flat Euclidean spaces are totally balanced according to example (2) in 3.13 without having a finite limit for $q_{w}(r)$. However, we do have the following

Corollary 5.3. Suppose that the $w$-model space $M_{w}^{m}$ is totally balanced and that 0 is not an asymptotic limit for the mean curvature function $\eta_{w}(R)$. Then $q_{w}(R)$ has a finite limit $q_{w}(\infty)$ for $R \rightarrow \infty$.

Proof. Since balance from above means that $q_{w}^{\prime}(r) \geq 0$, either $q_{w}(R)$ has a well-defined finite limit for $R \rightarrow \infty$ or $q_{w}(R)$ is increasing to $\infty$. In the latter case we apply the total balancing conditions in the form

$$
\frac{1}{q_{w}(R) m} \leq \eta_{w}(R) \leq \frac{1}{q_{w}(R)(m-1)}
$$

which then implies that $\eta_{w}(R) \rightarrow 0$ for $R \rightarrow \infty$. This contradicts the mean curvature assumption and shows the corollary.

The proof of corollary 2.3 now follows directly from the main theorem together with the observation that $S(R)$ may replace $R$ in the limit construction in the model space, where $\operatorname{Vol}\left(D_{R}\right)=\operatorname{Vol}\left(B_{S(R)}^{w}\right)$. The finiteness of the limit of $q_{w}$ follows from corollary 5.3. 


\section{Proof of Corollary 2.4}

Corollary 2.4 is not a direct consequence of Theorem 2.1, but rather a corollary of its proof and the proofs of Theorems A and B, applied to extrinsic balls when we suppose that $P^{m}=N^{n}$, so that the extrinsic distance becomes the intrinsic distance of $N$, and the extrinsic balls become intrinsic (geodesic) balls.

We give a sketch of the proof of the inequalities (3.19), (3.23) and (3.24) of Theorems A and B in this setting.

In fact, when $P^{m}=N^{n}$, we have that equality $\left\|\nabla^{P} r\right\|=1$ is fulfilled. Then, if $E_{R}^{w}(r)$ denotes the (radial) solution of the problem (3.20) defined now on $B_{R}^{w} \subseteq M_{w}^{n}$, and we transplant it to $B_{R}^{n} \subseteq N$, we have, applying inequality (3.18) to this transplanted function $E_{R}^{w}(r)$, and taking into account that $E_{R}^{w^{\prime}}(r) \leq 0$ for all $r \leq R$ and that we may formally put $H_{P}=0($ since $P=N)$,

$$
\Delta^{N} E_{R}^{w} \leq E_{R}^{w^{\prime \prime}}(r)+(n-1) E_{R}^{w^{\prime}}(r) \eta_{w}(r)=\Delta^{N} E_{R}
$$

where $E_{R}$ is the solution of

$$
\Delta^{N} E+1=0 \text { on } \quad B_{R}^{n}
$$

$$
\left.E\right|_{\partial B_{R}^{n}}=0
$$

Therefore, since $E_{R}^{w}(R)=E_{R}(R)=0$, the Maximum Principle gives

$$
E_{R}(x) \leq E_{R}^{w}(r(x)) \quad \text { for all } \quad x \in B_{R}^{n}
$$

Equality in this equation for some $x \in B_{R}^{n}$ implies equality for all $x$. The situation is then highly rigid in the following sense, which stems directly from equality in the Hessian comparison in Theorem 3.19: The radial sectional curvatures $K_{p, N}(x)$ must be equal to the model space 
radial curvatures $K_{w}(r(x))$ for all $x \in B_{R}^{n}$ and the corresponding Jacobi fields issuing from the pole $p$ must be scalar modifications of parallel fields. Thus the exponential map from $p$ generates an isometry from $B_{R}^{n}$ onto $B_{R}^{w}$

We also remark here that since $\left\|\nabla^{P} r\right\|=1$, the sign of $E_{R}^{w^{\prime \prime}}(r)-$ $E_{R}^{w^{\prime}}(r) \eta_{w}(r)$ is obsolete in this setting - the inequality (6.1) holds independent of this sign. Thence we do not, strictly speaking, need the condition that the $w$-model space be balanced from below. The reason for assuming total balance of the model space anyways is simply to guarantee that there is sufficient room for the symmetrization construction, cf. remark 3.15 .

From inequality (6.1) we obtain the isoperimetric inequality 3.23 of Theorem B, but now for geodesic balls

$$
\frac{\operatorname{Vol}\left(S_{R}^{n-1}\right)}{\operatorname{Vol}\left(B_{R}^{n}\right)} \geq \frac{\operatorname{Vol}\left(S_{R}^{w}\right)}{\operatorname{Vol}\left(B_{R}^{w}\right)} \text { for all } R>0,
$$

and, as a corollary using the arguments from $[\mathrm{Pa}]$ and from the proof of Theorem B,

$$
\operatorname{Vol}\left(B_{R}^{n}\right) \geq \operatorname{Vol}\left(B_{R}^{w}\right)
$$

Again, and for the same reason as above, equality in (6.5) is attained if and only if $B_{R}^{n}$ is isometric to the model space $R$-ball $B_{R}^{w}$.

Now, the further argument for corollary 2.4 follows the lines of the proof of Theorem 2.1: We have that

$$
\mathcal{A}_{1}\left(B_{R}^{n}\right)=\int_{B_{R}^{n}} E_{R} \leq \int_{B_{R}^{n}} E_{R}^{w} \leq \int_{B_{R}^{n *}} E_{R}^{w *} .
$$


where $B_{R}^{n *}=B_{S(R)}^{w}$ is the Schwarz symmetrization of the geodesic ball $B_{R}^{n}$ in the $w$-model space $M_{w}^{n}$, i.e., the geodesic ball $B_{S(R)}^{w} \subseteq M_{w}^{n}$ such that $\operatorname{Vol}\left(B_{R}^{n}\right)=\operatorname{Vol}\left(B_{S(R)}^{w}\right)$. We know that $S(R) \geq R$, from (6.5).

Now consider the radial solution $E_{S(R)}^{w}(r)$ of the problem

$$
\begin{aligned}
\Delta^{M_{w}^{n}} E & =-1 \text { on } B_{S(R)}^{w} \\
\left.E\right|_{\partial B_{S(R)}^{w}} & =0
\end{aligned}
$$

With a totally analogous argument as in the proof of Theorem 2.1, using the sets $D(\mu)=\left\{x \in B_{R}^{n} \mid E_{R}^{w}(r(x)) \geq \mu\right\}$, which are geodesic balls in $N$ with radius $g(\mu)=E_{R}^{w^{-1}}(\mu)$, we conclude that

$$
E_{R}^{w *}(t) \leq E_{S(R)}^{w}(t) \text { for all } t \in[0, S(R)],
$$

So

$$
\mathcal{A}_{1}\left(B_{R}^{n}\right) \leq \int_{B_{R}^{n *}} E_{R}^{w *} d \sigma \leq \int_{B_{S(R)}^{w}} E_{S(R)}^{w} d \sigma=\mathcal{A}_{1}\left(B_{S(R)}^{w}\right)
$$

Equality in (2.3) has in particular the consequence, stemming from the equality discussion inserted into the codimension-0 discussion of Theorems A and B above, that the exponential map from the pole $p$ generates directly an isometry from $B_{R}^{n}$ onto $B_{R}^{w}$ as claimed. In particular, therefore, $S(R)=R$, and this finishes the proof of corollary 2.4 .

\section{REFERENCES}

[Ba] C. Bandle, Isoperimetric Inequalities and Applications, Pitman Publishing Inc. (1980). 
[BBC] R. Bañuelos, M. van den Berg, and T. Carroll, Torsional rigidity and expected lifetime of Brownian motion, J. London Math. Soc.,(2) 66 (2002), 499-512.

[Ber] P. H. Berard, Spectral Geometry: Direct and Inverse Problems, Lecture Notes in Mathematics Vol. 1207, Springer -Verlag (1986).

[BG] M. van den Berg and P. B. Gilkey, Heat content and Hardy inequality for complete Riemannian manifolds, Bull. Lond. Math. Soc. 36 (2004), $577-586$.

[Ch1] I. Chavel, Eigenvalues in Riemannian Geometry, Academic Press (1984).

[Ch2] I. Chavel, Isoperimetric inequalities. Differential geometric and analytic perspectives, Cambridge Tracts in Mathematics, 145. Cambridge University Press (2001).

[CLY] S. Y. Cheng, P. Li, and S. T. Yau, Heat equations on minimal submanifolds and their applications, Amer. J. Math. 106 (1984), 1033-1065.

[Dy] E. B. Dynkin Markov processes, Springer Verlag (1965).

[GreW] R. Greene and H. Wu, Function theory on manifolds which possess a pole, Lecture Notes in Math., vol. 699, Springer-Verlag, Berlin and New York (1979).

[Gri] A. Grigor'yan, Analytic and geometric background of recurrence and non-explosion of the Brownian motion on Riemannian manifolds, Bull. Amer. Math. Soc. 36 (1999), 135-249.

[H] R. Z. Ha'sminskii, Probabilistic representation of the solution of some differential equations, in Proc. 6th All Union Conf. on Theor. Probability and Math. Statist. (Vilnius 1960), (1960)

[JK] L. P. Jorge and D. Koutroufiotis, An estimate for the curvature of bounded submanifolds, Amer. J. Math. 103 (1981), 711-725.

[KP] L. Karp and M. Pinsky, Volume of a small extrinsic ball in a submanifold, Bull. London Math. Soc. 21 (1989), 87-92.

[Ma1] S. Markvorsen, On the mean exit time from a minimal submanifold, J. Diff. Geom. 29 (1989), 1-8 .

[Ma2] S. Markvorsen, On the heat kernel comparison theorems for minimal submanifolds, Proc. Amer. Math. Soc. 97 (1986), 479-482. 
[MP1] S. Markvorsen and V. Palmer, Generalized isoperimetric inequalities for extrinsic balls in minimal submanifolds, J. reine angew. Math., 551, (2002), 101-121.

[MP2] S. Markvorsen and V. Palmer, Transience and Capacity of Minimal submanifolds, GAFA, Geom. funct. anal. 13, (2003), 915-933.

[MP3] S. Markvorsen and V. Palmer, How to obtain Transience from bounded radial mean curvature, Trans. Amer. Math. Soc. 357, (2005),3459-3479.

[Mc] P. McDonald, Isoperimetric conditions, Poisson problems, and diffusions in Riemannian manifolds, Potential Analysis 16, (2002), 115-138.

[O'N] B. O'Neill, Semi-Riemannian Geometry; With Applications to Relativity, Academic Press (1983).

[Os] R. Osserman, The Isoperimetric Inequality, Bull. Amer. Math. Soc. (N.S) 84, (2) (1978), 1182-1238

[Pa] V. Palmer, Isoperimetric Inequalities for extrinsic balls in minimal submanifolds and their applications, Jour. London Math. Soc. 60, (2) (1999), 607-616.

[PS] G. Pólya and G. Szegö, Isoperimetric Inequalities in Mathematical Physics, Princeton University Press (1951).

[Po] G. Pólya, Torsional rigidity, principal frequency, electrostatic capacity and symmetrization, Quart. Appl. Math. 6, (1948), 267-277.

[S] T. Sakai, Riemannian Geometry, Translations of Mathematical Monographs Volume 149, American Mathematical Society, 1996.

Department of Mathematics, Technical University of Denmark.

E-mail address: S.Markvorsen@mat.dtu.dk

Departament de Matemàtiques, Universitat Jaume I, Castellon, Spain.

E-mail address: palmer@mat.uji.es 